\title{
An Atypical Case of Mild COVID-19 Infection with Severe Guillain-Barré Syndrome as Neurological Manifestation
} \author{
Saswat Subhankar ${ }^{1}$ Vikram Samal $^{2}$ \\ ${ }^{1}$ Department of Pulmonary, Critical Care and Sleep Medicine, KIMS, \\ Bhubaneswar, Odisha, India \\ ${ }^{2}$ Department of Paediatrics, SCB Medical College, Cuttack, Odisha, \\ India \\ ${ }^{3}$ Acharya Harihar Post Graduate Institute of Cancer, SCB Medical \\ College, Cuttack, Odisha, India \\ ${ }^{4}$ Department of Pulmonary Medicine, K.S. Hegde Medical Academy, \\ Mangalore, Karnataka, India
}

Debasis Behera ${ }^{1}$ Ruchi Rekha Behera² Suman Kumar Jagaty ${ }^{1}$ Rekha Das ${ }^{3}$ Rajesh Venkataram ${ }^{4}$

\begin{abstract}
Address for correspondence Debasis Behera, MD, Department of Pulmonary, Critical Care and Sleep Medicine, KIMS, Bhubaneswar, Odisha 751024, India (e-mail: drdebasis8@gmail.com).
\end{abstract}

J Health Allied Sci NU 2022;12:93-95.

\begin{abstract}
Introduction More than 80 million people have been infected with coronavirus disease 2019 (COVID-19) infection worldwide till date with more than 17,00000 fatalities. Although COVID-19 commonly affects respiratory system in the form of cough and dyspnea, a neurotropic presentation has been described in one-third of patients.

Objective We report an atypical case of COVID-19 with mild symptoms who presented to our hospital with features suggestive of severe Guillain-Barré syndrome (GBS). Discussion The mechanisms by which severe acute respiratory syndrome coronavirus 2 causes neurologic damage are multifactorial, including direct damage to specific receptors, cytokine-mediated injury, secondary hypoxia, and retrograde travel along nerve fibers. The pathogenesis of GBS secondary to COVID-19 is not yet well understood. It is hypothesized that viral illnesses-related GBS could be mediated due to autoantibodies or direct neurotoxic effects of viruses.

Keywords

- COVID-19

- GBS

- AMSAN

- lung collapse

- bronchoscopy

Conclusion In this ongoing era of pandemic, it is very important for the clinicians to be aware of association of GBS with COVID-19, as early diagnosis and treatment of this complication could have gratifying results. It is also very important to differentiate GBS from critical illness neuropathy and respiratory distress secondary to COVID-19 itself, as treatment to the above conditions is quite different and inability to correctly diagnose could lead to significant increase in morbidity and mortality.
\end{abstract}

\section{Introduction}

More than 80 million people have been infected with coronavirus disease 2019 (COVID-19) infection worldwide till date with more than 17,00000 fatalities. ${ }^{1}$ Although COVID-19 commonly affects respiratory system in the form

published online Jun 08, 2021
DOI https://doi.org/

10.1055/s-0041-1731142

ISSN 2582-4287 of cough and dyspnea, a neurotropic presentation has been described in one-third of patients. ${ }^{2}$ Some of these neurological symptoms have proved to be quite specific, for example, loss of smell or taste, but other ones are nonspecific, for example, headache, dizziness, or reduced level of
(C) 2021. Nitte (Deemed to be University).

This is an open access article published by Thieme under the terms of the Creative Commons Attribution-NonDerivative-NonCommercial-License, permitting copying and reproduction so long as the original work is given appropriate credit. Contents may not be used for commercial purposes, or adapted, remixed, transformed or built upon. (https://creativecommons.org/licenses/by-nc-nd/4.0/).

Thieme Medical and Scientific Publishers Pvt. Ltd. A-12, 2nd Floor,

Sector 2, Noida-201301 UP, India 
consciousness. $^{3}$ However, whether the neurological symptoms associated with severe acute respiratory syndrome coronavirus 2 are attributable to secondary mechanisms (i.e., multiorgan dysfunction or systemic inflammation), an abnormal immune response or the direct injury of the virus is still unknown. ${ }^{4}$ Guillain-Barré syndrome (GBS) is best described as an acute inflammatory polyradiculoneuropathy clinically characterized by areflexia and progressive weakness of arms and legs. Though many rare variants of GBS have been described, the commonly observed subtypes are acute motor axonal neuropathy, acute motor sensory axonal neuropathy, and acute inflammatory demyelinating polyradiculoneuropathy. ${ }^{5}$ We report a case of post-COVID-19 patient, mild variety, who presented to our hospital with features suggestive of severe GBS.

\section{Case Presentation}

Our patient was a 68-year-old woman with a medical history significant for hypertension for the last 5 years and was on regular treatment. Two weeks prior to this hospital admission, she developed a fever, cough, and myalgia amidst the COVID-19 pandemic. The patient was tested positive for COVID-19 by reverse transcription polymerase chain reaction test and was treated with doxycycline, ivermectin, multivitamin, and paracetamol for 5 days at home. Subsequently, over the next 2 weeks, her fever and cough resolved; however, she developed new onset of numbness, pins, and needles sensation in the lower extremity associated with mild weakness. These same symptoms then progressed to the upper extremity over the next 24 hours with an unstable gait. She had no history of headaches, loss of consciousness, changes in mental status, changes in vision and speech, difficulty swallowing, seizure, or urine/bowel incontinence.

She denied any history of insect bites or trauma. Physical examination findings included stable vital signs and motor strength was $4 / 5$ in all four extremities, diminished deep tendon reflexes in both upper and lower extremities, and a negative Babinski sign bilaterally. There was no clear-cut spine sensory level and no upper motor neuron weakness findings. She was admitted to the hospital for further evaluation and management.

\section{Investigations}

On the day of admission, notable laboratory findings were a white cell count of $12.6 \times 109 / \mathrm{L}$, raised D-dimer $(1.84 \mu \mathrm{g} / \mathrm{mL})$ with normal erythrocyte sedimentation rate, C-reactive protein, lactate dehydrogenase, ferritin, vitamin B12 level, and thyroid-stimulating hormone. The magnetic resonance imaging (MRI) scan of the head showed no intraparenchymal mass or lesions, no hemorrhage, and no findings suggestive of hydrocephalus. MRI of the spine was unremarkable. Repeat COVID-19 testing was negative. The patient developed worsening of her weakness with muscle strength gradually decreased to $2 / 5$ in both the lower and upper extremity. With the patient exhibiting ascending weakness with diminished reflexes, an urgent lumbar puncture was performed to rule out GBS. Cerebrospinal fluid (CSF) revealed findings suggestive of albumin-cytologic dissociation with a white cell count of $2 \mathrm{cu} / \mathrm{mm}$ and protein count of $101 \mathrm{mg} / \mathrm{dL}$. Nerve conduction study was performed suggestive of demyelinating axonal sensory motor polyradiculoneuropathy. Hence, a diagnosis of post-COVID GBS was made based on the history, clinical examination, and investigations.

\section{Hospital Course}

However, patient condition was gradually deteriorated and she was intubated because of respiratory muscle weakness as evidenced by gradual decreased in single breath count with decreased maximal inspiratory pressure and maximal expiratory pressure. Intravenous immunoglobulin (IVIG) was initiated as per neurologist opinion along with other supportive measures. In the meantime, there was collapse of right lung because of mucous plugging, and positional therapy along with chest physiotherapy was given. On the next day, right lung was expanded; however, the left lung was collapsed and did not expand in spite of conservative treatment. Therapeutic bronchoscopy was performed for mucous plug removal resulting in complete expansion of bilateral lungs. In view of prolonged mechanical ventilation, tracheostomy was performed on day 8. Endotracheal aspirate culture was showing growth of pseudomonas; appropriate antibiotics were initiated as per culture report. Aggressive physical therapy for chest and muscle was given. With above-mentioned treatment, she was improved gradually and decannulated on day 20 and subsequently discharged in stable condition.

\section{Discussion}

Neurologic manifestations of COVID-19 are increasing, but very few cases of GBS associated with this virus have been established. GBS is an immune-mediated response, likely from a recent infection, where the immune system affects the peripheral nerves due to a molecular mimicry phenomenon. This has preceded two-thirds of the times by an upper respiratory infection or gastroenteritis. The case series by Mao et al in Wuhan, China, was one of the first studies that showed neurologic involvement in patients with COVID-19. They concluded that patients with more severe COVID-19 infection were more likely to have neurologic symptoms. ${ }^{6}$ In contrast, our patient's respiratory status was relatively stable during the initial part of COVID-19 infection; however, later during GBS she landed up in respiratory complications leading to need of mechanical ventilation and frequent lung collapse leading to delay in recovery.

In Italy, a series of five patients were diagnosed with GBS 5 to 10 days after a viral illness from COVID-19. Similar to our patient, they did not show typical MRI findings of GBS including surface thickening and contrast enhancement on the conus medullaris and the nerve roots of the cauda equina. Only one of the five patients had a functional recovery to the point of ambulation. ${ }^{7}$

The literature shows there is variability in the presentation of COVID-19 and GBS. Our case had a typical course of 
COVID-19 symptoms preceding GBS findings. However, two other case reports identified concurrent respiratory and neurologic symptoms. Besides, the duration from onset of viral illness to neurologic manifestations has ranged from 5 to 24 days. In all cases reported, treatment with IVIG was administered. However, the recovery varied from full neurologic recovery to no change in extremity function and terminal respiratory failure. ${ }^{8-10}$

There are several theories on how the virus attacks the nervous system. Studies postulate that the virus can infect a peripheral neuron, use an active retrograde transport mechanism across the synapse onto the cell body, and reach the brain. Other proposed mechanisms include direct damage through angiotensin-converting enzyme-2 receptors, cytokine-related injury, and hypoxia-related sequela. It is unclear if the COVID-19 itself triggers the formation of antibodies against any specific forms of glycolipids seen in some forms of GBS.,11 Although our patients had positive polymerase chain reaction throat swab test, their CSF examination did not show any raised cell count and contrast MRI of the spine did not show any enhancement of caudal nerve roots, thus favoring immune mediated hypothesis. This was further strengthened by an excellent response to IVIG, thus favoring an immune-mediated pathogenesis rather than direct viral damage. There is a need for further investigation into how COVID-19 is related to GBS.

\section{Conclusion}

Nervous system involvement in COVID-19 may have been grossly underestimated. In this ongoing era of pandemic, it is very important for the clinicians to be aware of association of GBS with COVID-19, as early diagnosis and treatment of this complication could have gratifying results. It is also very important to differentiate GBS from critical illness neuropathy and respiratory distress secondary to COVID-19 itself, as treatment to the above conditions is quite different and inability to correctly diagnose could lead to significant increase in morbidity and mortality.

\section{Authors' Contributions}

All the authors have contributed substantially to the conception or design of the work; the acquisition, analysis, or interpretation of data for the work; drafting and revision of the work critically for important intellectual content; final approval of the version to be published; agreement to be accountable for all aspects of the work in ensuring that questions related to the accuracy or integrity of any part of the work are appropriately investigated and resolved.

\section{Conflict of Interest}

None declared.

\section{References}

1 Coronavirus Disease (COVID-19) Situation Reports. Who. int. Available at: https://www.who.int/emergencies/diseases/ novel-coronavirus-2019/situation-reports. Published 2021. Accessed May 16, 2021.

2 Carrillo-Larco RM, Altez-Fernandez C, Ravaglia S, Vizcarra JA. COVID-19 and Guillain-Barre syndrome: a systematic review of case reports. Wellcome Open Res 2020;5:10710.12688/ wellcomeopenres.15987.2

3 Politi LS, Salsano E, Grimaldi M. Magnetic resonance imaging alteration of the brain in a patient with coronavirus disease 2019 (COVID-19) and anosmia. JAMA Neurol 2020;77(8):1028-1029

4 Bridwell R, Long B, Gottlieb M. Neurologic complications of COVID-19. Am J Emerg Med 2020;38(7):1549.e3-1549.e7

5 Piccione EA, Salame K, Katirji B, Guillain-Barré syndrome and related disorders. In: Katirji B, Kaminski H, Ruff R, eds. Neuromuscular Disorders in Clinical Practice. New York, NY: Springer; 2014

6 Mao L, Jin H, Wang M, et al. Neurologic manifestations of hospitalized patients with coronavirus disease 2019 in Wuhan, China. JAMA Neurol 2020;77(6):683-690

7 Toscano G, Palmerini F, Ravaglia S, et al. Guillain-Barré syndrome associated with SARS-CoV-2. N Engl J Med 2020;382(26):2574-2576

8 Alberti P, Beretta S, Piatti $\mathrm{M}$, et al. Guillain-Barré syndrome related to COVID-19 infection. Neurol Neuroimmunol Neuroinflamm 2020;7(4):e74110.1212/ nxi.0000000000000741

9 Virani A, Rabold E, Hanson T, et al. Guillain-Barré syndrome associated with SARS-CoV-2 infection. IDCases 2020;20:e007 7110.1016/j.idcr.2020.e00771

10 Camdessanche JP, Morel J, Pozzetto B, Paul S, Tholance Y, Botelho-Nevers E. COVID-19 may induce Guillain-Barré syndrome. Rev Neurol (Paris) 2020;176(6):516-518

11 Baig AM, Khaleeq A, Ali U, Syeda H. Evidence of the COVID-19 virus targeting the CNS: tissue distribution, host-virus interaction, and proposed neurotropic mechanisms. ACS Chem Neurosci 2020;11(7):995-998 